\title{
Atuação dos enfermeiros ante aos direitos dos pacientes: tomada de decisão, identidade e autonomia pessoal
}

Andreia Alves Ramos ${ }^{1}$, Luciana Neves da Silva Bampi ${ }^{2}$, Valéria Lerch Lunardi ${ }^{3}$

\section{RESUMO}

Estudo que objetivou verificar a percepção dos enfermeiros acerca de sua atuação ante os direitos dos pacientes, considerando os fundamentos: tomada de decisão, identificação do paciente e autonomia pessoal. Estudo quantitativo, descritivo-observacional e transversal, realizado num Hospital Universitário de Brasília-DF. Dados foram coletados a partir da aplicação de instrumento específico para o objetivo proposto já validado, a 128 enfermeiros. Realizada análise estatística descritiva e teste estatístico não-paramétrico de Wilcoxon. Verificou-se que os enfermeiros perceberam-se atuando em defesa dos direitos dos pacientes em todos os fundamentos analisados sendo a autonomia pessoal o mais exercido e o favorecimento de informações para a tomada de decisão como o menos exercido. $O$ estudo viabilizou a verificação de quais fundamentos do respeito aos direitos dos pacientes são percebidos como mais respeitados, além de produzir questionamento acerca do conhecimento teórico que os enfermeiros possuem sobre os conceitos e a aplicação destes fundamentos.

Descritores: Ética em Enfermagem; Direitos dos Pacientes; Autonomia Pessoal; Bioética.

\footnotetext{
${ }^{1}$ Enfermeira, Mestre em Enfermagem. Enfermeira da Rede Sarah Hospitais de Reabilitação. Brasília, DF, Brasil. E-mail: ramos.andreiaalves@gmail.com.

${ }^{2}$ Enfermeira, Doutora em Ciências da Saúde. Professora Adjunto do Departamento de Enfermagem da Universidade de Brasília. Brasília, DF, Brasil. E-mail: lbampi@unb.br.

${ }^{3}$ Enfermeira, Doutora em Enfermagem. Professora Associada da Escola de Enfermagem da Universidade Federal do Rio Grande. Rio Grande, RS, Brasil. E-mail: vlunardi@terra.com.br.
}

Artigo recebido: 24/03/2018. Artigo aprovado: 21/11/2018. Artigo publicado: 31/12/2018.

\section{Como citar esse artigo:}

Ramos AA, Bampi LNS, Lunardi VL. Atuação dos enfermeiros ante aos direitos dos pacientes: tomada de decisão, identidade e autonomia pessoal. Rev. Eletr. Enf. [Internet]. 2018 [acesso em: ];20:v20a54.

Disponível em: https://doi.org/10.5216/ree.v20.52105. 


\section{INTRODUÇÃO}

Os avanços no conhecimento científico e tecnológico têm marcado as sociedades mais desenvolvidas, repercutindo nas mais diversas áreas, entre elas a saúde ${ }^{(1)}$. É inegável que tais avanços contribuíram para salvar vidas, revolucionando a qualidade de vida e a longevidade humana ${ }^{(2)}$, entretanto, conferiram aos profissionais de saúde um poder intervencionista jamais imaginado. Nesse cenário, os doentes passaram a reivindicar que suas opiniões fossem consideradas no processo de tomada de decisão clínica. Essa atitude impulsionou uma mudança na relação clínica, tradicionalmente vertical e infantilizada, paternalista, na qual o paciente obedece ao médico, para uma nova relação, mais horizontal e democrática, na qual a participação do doente é ativa e questionadora(3): a autonomista.

O discurso sobre autonomia se assenta na modernidade, no reconhecimento da dignidade humana e da capacidade que a razão e a vontade conferem a pessoa para realizar escolhas livres, para autodeterminar-se ${ }^{(4)}$. A Carta de Direitos dos Pacientes, de 1972, criada nos Estados Unidos ${ }^{(5)}$, foi um dos primeiros instrumentos legais que contemplou a autonomia como atributo do ser humano doente, considerando-o capaz de decidir livremente sobre si, a partir da racionalização das informações sobre sua situação clínica, possíveis opções de escolha e valores envolvidos.

A Bioética tem se firmado, desde meados do século passado, como disciplina capaz de auxiliar na discussão sobre a conduta humana nas áreas das ciências da saúde e da vida, uma vez que a interpreta à luz de valores e princípios morais ${ }^{(4)}$. As preocupações com os aspectos éticos na prestação de cuidados de saúde não se reduzem às normas contidas na legislação ou nos códigos de ética profissional, mas compreendem o respeito à pessoa como ser social, admitindo que sua essência é a liberdade, com compromisso e responsabilidade ${ }^{(6)}$.

A Enfermagem, como a Bioética, também incorpora os conceitos de dignidade humana e autonomia para definir o cuidado numa perspectiva ética ${ }^{(4)}$. Atualmente, a essência ética do cuidado de enfermagem pode ser definida como: prover o cuidado em resposta à vulnerabilidade do ser humano com o objetivo de manter, proteger e promover a dignidade o máximo possível ${ }^{(7)}$. Respeitar e promover a autonomia do paciente é um exercício que requer a realização de pressupostos já definidos na literatura Bioética: "dizer a verdade; respeitar a privacidade; proteger informações confidenciais; obter consentimento para intervenções e, quando solicitado, ajudar na tomada de decisões"(8).

Apesar disso, a literatura refere que a consideração do princípio do respeito a autonomia em Enfermagem é um tema polêmico e um importante problema para a Bioética(4). Os enfermeiros, principalmente no ambiente hospitalar, têm dificuldade de colocar em prática o princípio da autonomia por diferentes motivos, seja pela falta de clareza sobre o significado e alcance do princípio; seja pelo equivocado entendimento de que exista divergência entre o princípio da autonomia e a obrigação de cuidar ${ }^{(4)}$. Existe responsabilidade por parte dos enfermeiros em promover a dignidade e autonomia dos doentes por meio da interação destes com a equipe de saúde ${ }^{(9)}$. Essa responsabilidade está elencada no Código de Ética dos Profissionais de Enfermagem no rol de deveres: "respeitar o exercício da autonomia da pessoa ou do representante legal na tomada de decisão"(10).

Diante do exposto, fica clara a importância ética, moral e deontológica dos enfermeiros desenvolverem ações que permitam o respeito e a promoção da autonomia e dos direitos dos pacientes na prática assistencial. Entretanto, não está claro se esses profissionais estão aptos a realizá-las ${ }^{(4)}$, ou se percebem-se atuando no sentido 
de implementá-las. Um estudo brasileiro ${ }^{(11)}$ apresentou um instrumento capaz de verificar se os enfermeiros estavam atuando ante a defesa dos direitos dos pacientes a partir das suas próprias percepções. Essa ferramenta contribui para o estudo do tema autonomia do paciente.

Utilizar esse instrumento ${ }^{(11)}$ para aprofundar os conhecimentos sobre a prática cotidiana dos enfermeiros, no sentido de identificar posturas que possam contribuir ou não com o respeito à autonomia e aos direitos dos pacientes torna-se essencial ao abordar a relevância e a responsabilidade dos enfermeiros na assistência em saúde. Além disso, já é reconhecido que tratamentos e cuidados em saúde quando são qualificados como respeitosos e dignos pelos doentes, têm potencial de apresentar melhores resultados ${ }^{(9)}$.

Os resultados da presente pesquisa poderão compor o referencial teórico de respeito a autonomia do paciente, a partir de cuidados de enfermagem. Nessa perspectiva, a pesquisa foi desenvolvida com o objetivo de verificar se os enfermeiros se percebem respeitando os direitos dos pacientes na prática assistencial hospitalar, considerando os fundamentos: tomada de decisão, identidade e autonomia pessoal.

\section{MÉTODO}

Estudo de abordagem quantitativa com método descritivo-observacional com corte de tempo transversal. Pesquisa realizada num Hospital Universitário de Brasília-DF. A coleta de dados ocorreu de junho a dezembro de 2016. Participaram da pesquisa 128 enfermeiros. O cálculo amostral foi definido por amostragem aleatória simples, considerando um total de 250 enfermeiros, os quais exerciam suas atividades laborais em setores assistenciais de internação, ambulatório e diagnóstico a pacientes adultos. 0 erro amostral considerado foi de $5 \%$.

Foram utilizados dois instrumentos para a coleta de dados. O primeiro, consiste em um instrumento específico desenvolvido e validado ${ }^{(11)}$ para verificar a "percepção dos enfermeiros acerca da sua atuação ante os direitos dos clientes". Este instrumento é constituído por 13 questões com respostas operacionalizadas em escala Likert, variando de 1, nunca, a 5, sempre. A validação do instrumento, foi verificada através dos testes de análise fatorial e o Alfa de Cronbach. A análise fatorial agrupou as questões do instrumento em quatro diferentes construtos, representando diferentes facetas percebidas quanto aos direitos ${ }^{(11)}$. Os construtos propostos foram definidos conceitualmente como: preservação da individualidade, compreendendo ações implementadas para assegurar o respeito à intimidade e às características individuais do paciente; respeito à autonomia do cliente, que implica o direito do cliente de autodeterminar-se; identificação pessoal, referindo-se ao direito do cliente de ser identificado individualmente quanto de poder identificar pessoalmente os profissionais com quem se relaciona; e, informação para a tomada de decisão, como o direito do cliente de ser informado, de modo a subsidiar as tomadas de decisões ${ }^{(11)}$.

Um segundo instrumento, contendo perguntas relacionadas a sexo, idade, tempo de formação, formação em pós-graduação, tempo de serviço e local de desenvolvimento das atividades no Hospital estudado foi utilizado para caracterizar a amostra estudada. Os instrumentos de coleta de dados foram autoadministrados e para orientação sobre o preenchimento havia uma breve instrução na folha de apresentação.

Os dados foram analisados utilizando o programa IBM Statistical Package for Social Sciences (SPSS), versão 24.0. As variáveis consideradas na análise dos dados foram: idade, sexo, tempo de formação, ter pós-graduação, tempo de trabalho na instituição, unidade de atuação no Hospital estudado e presença de outro vínculo 
Ramos AA, Bampi LNS, Lunardi VL.

empregatício. A caracterização da amostra foi realizada por análise descritiva, utilizando as frequências absoluta e relativa. A variável dependente do estudo - percepção do enfermeiro acerca de sua atuação ante aos direitos dos pacientes - foi mensurada a partir dos quatro construtos propostos no instrumento citado ${ }^{(11)}$ : preservação da individualidade, respeito à autonomia do cliente, identificação pessoal e informação para a tomada de decisão. Foram calculadas médias e desvios-padrão dos escores obtidos na aplicação do instrumento por constructo. Os valores médios de cada constructo foram comparados entre si. Para tal, foi utilizada a análise estatística não paramétrica. Aplicou-se o teste de Wilcoxon para comparação de duas amostras não pareadas, considerando $p>0,001$. A partir desta comparação é possível constatar qual constructo da variável dependente foi mais considerado em relação a outro.

O respeito aos requisitos éticos foi orientado pela Resolução do Conselho Nacional de Saúde no 466 de 2012. O projeto foi apreciado pelo Comitê de Ética em Pesquisa da Faculdade de Ciências da Saúde, da Universidade de Brasília, obtendo aprovação em abril de 2016 (parecer 1.484.752). O aceite em participar da pesquisa foi confirmado pelo participante mediante a assinatura do Termo de Consentimento Livre e Esclarecido (TCLE).

\section{RESULTADOS}

A amostra estudada foi composta em sua maioria por mulheres (83\%) com idade inferior a 40 anos (83\%). Os participantes, de forma majoritária, apresentavam tempo de formação profissional inferior a 10 anos (73\%), tendo como maior titulação referida a pós-graduação lato-sensu (83,6\%). Predominantemente, os enfermeiros atuavam em unidades de internação hospitalar (77,3\%), com tempo de serviço na instituição entre dois a quatro anos (46,9\%) e com vínculo empregatício apenas com a instituição na qual foi desenvolvida a pesquisa (63,3\%).

A avaliação da percepção dos enfermeiros quanto ao seu respeito aos direitos dos pacientes foi realizada a partir da aplicação de instrumento específico ${ }^{(11)}$. O instrumento divide-se em quatro dimensões e/ou constructos que representam os fundamentos básicos do respeito aos direitos dos doentes. Considerando que a variação de respostas na forma de escala Likert do instrumento foi de 1 (nunca), 2 (raramente), 3 (às vezes), 4 (na maioria das vezes) e 5 (sempre). A Tabela 1 apresenta a tendência central e a dispersão dos escores obtidos com a aplicação do instrumento. 
Tabela 1: Média e desvio-padrão por questão e por constructo do instrumento - Percepção do enfermeiro quanto ao seu respeito aos direitos dos pacientes. Brasília, DF, Brasil, 2017.

\begin{tabular}{|c|c|c|c|}
\hline & $\mathbf{N}$ & Média & DP \\
\hline Informação para a tomada de decisão & & 4,07 & 0,27 \\
\hline Forneço ao cliente informações compreensíveis sobre o nome dos medicamentos administrados & 128 & 4,35 & 0,84 \\
\hline $\begin{array}{l}\text { Forneço ao cliente informações compreensíveis sobre possíveis benefícios da terapêutica instituída } \\
\text { durante a sua internação hospitalar }\end{array}$ & 128 & 4,31 & 0,68 \\
\hline $\begin{array}{l}\text { Forneço ao cliente informações compreensíveis sobre os possíveis efeitos dos medicamentos } \\
\text { administrados }\end{array}$ & 128 & 4,07 & 0,84 \\
\hline $\begin{array}{l}\text { Forneço ao cliente informações compreensíveis sobre possíveis riscos da terapêutica instituída durante } \\
\text { a sua internação hospitalar }\end{array}$ & 128 & 3,88 & 0,95 \\
\hline $\begin{array}{l}\text { Forneço ao cliente informações compreensíveis sobre possíveis riscos das ações diagnósticas durante } \\
\text { a sua internação hospitalar }\end{array}$ & 128 & 3,72 & 1,00 \\
\hline Identificação pessoal & & 4,31 & 0,34 \\
\hline $\begin{array}{l}\text { Identifico-me ao cliente sob meus cuidados através de uma apresentação prévia (informando meu } \\
\text { nome, cargo e função) }\end{array}$ & 128 & 4,55 & 0,71 \\
\hline Chamo o cliente pelo nome e sobrenome & 128 & 4,07 & 1,07 \\
\hline Respeito à autonomia do cliente & & 4,64 & 0,03 \\
\hline Respeito o direito do cliente de consentir ou de recusar procedimentos de enfermagem nele realizados & 128 & 4,68 & 0,52 \\
\hline Respeito o direito do cliente de consentir ou de recusar ações terapêuticas a que será submetido & 128 & 4,62 & 0,69 \\
\hline Respeito o direito do cliente de ter acesso a seu prontuário & 128 & 4,63 & 0,80 \\
\hline
\end{tabular}

\section{DISCUSSÃO}

$\mathrm{Na}$ análise do constructo - Informação para a tomada de decisão - verifica-se percepção do enfermeiro acerca da sua atitude em fornecer informações compreensíveis ao doente para que o mesmo possa tomar uma decisão acerca dos cuidados em saúde. A tomada de decisão é o direito do paciente de decidir e eleger qual a melhor opção, dentre as existentes e possíveis, considerando suas próprias convicções e de acordo com seu próprio projeto de vida, sem que haja interferência de terceiros ${ }^{(8)}$. Fundamentada no princípio ético do respeito à autonomia, na tomada de decisão autônoma os indivíduos devem atuar intencionalmente, com conhecimento e sem influências externas que exerçam controle sobre suas ações ${ }^{(8)}$.

A média obtida neste constructo aponta que na maioria das vezes, os enfermeiros fornecem informações que subsidiam a tomada de decisão dos doentes, sendo essas informações em sua maioria relacionadas ao nome dos medicamentos administrados $(4,35)$, e, de forma minoritária, sobre os riscos das ações diagnósticas $(3,72)$.

Uma revisão sistemática ${ }^{(12)}$ identificou o papel do enfermeiro no processo de tomada de decisão no tratamento de pessoas com câncer. Fornecer informações ao paciente é um dos principais papéis do enfermeiro na prática clínica. Doentes, cuidadores e familiares identificaram os enfermeiros como fonte segura e confiável de informações, sobretudo quando se trata da terapêutica, das ações diagnósticas possíveis, da trajetória do tratamento e dos sintomas da doença, incluindo o prognóstico. A confiabilidade das informações fornecidas pelos enfermeiros advém, provavelmente, da estreita relação que o profissional desenvolve com o enfermo, devido ao grande número de horas diárias despendidas no cuidado ${ }^{(13)}$.

O Código de Ética dos profissionais de Enfermagem ratifica a obrigatoriedade do fornecimento de informações aos pacientes quando determina, em seu artigo 39, o dever profissional de "Esclarecer à pessoa, família e coletividade, a respeito dos direitos, riscos, benefícios e intercorrências acerca da assistência de Enfermagem", bem como, "Orientar sobre preparo, benefícios e consequências decorrentes de exames e outros procedimentos, respeitando o direito de recusa da pessoa ou de seu representante legal" (10). Apesar de se 
constituir como obrigatoriedade profissional, o fornecimento de informações para a tomada de decisão obteve médias menores nas questões em que o conteúdo tratava dos riscos da terapêutica instituída, das ações diagnósticas e dos medicamentos administrados. A omissão pelos enfermeiros de informações sobre os riscos inerentes ao tratamento ou a consideração apenas dos fatos benéficos da terapêutica sugerem que este profissional valoriza o princípio da beneficência em detrimento do princípio da autonomia da pessoa. Por conhecer a importância das ações terapêuticas, diagnósticas e medicamentosas, a atitude de omitir informações sustentase na ideia de que o doente poderia recusar submeter-se a elas caso tivesse ciência dos riscos e dos efeitos adversos conhecidos.

O papel do enfermeiro na tomada de decisão do paciente foi objeto de uma pesquisa internacional|(14), a qual verificou que estes profissionais atuam no processo educativo/informativo do doente esclarecendo o plano de tratamento repassado pelo médico, com linguagem mais acessível. Nesse estudo, a defesa do paciente, o compartilhamento de informações sobre o doente para a equipe multidisciplinar, os sintomas e os efeitos adversos da terapêutica instituída e o apoio psicológico foram reconhecidos como papéis do enfermeiro no processo de tomada de decisão compartilhada.

No Brasil, os enfermeiros ainda sentem-se enfraquecidos para atuar em situações que envolvam tomada de decisão do paciente, isto porque o poder decisório acerca da conduta terapêutica dos pacientes ainda está centralizado na equipe médica ${ }^{(15)}$. Existem barreiras, já identificadas na literatura, que limitam a participação do enfermeiro no processo de tomada de decisão ${ }^{(13)}$, sendo elas relacionadas à prática clínica, quando não existe padronização de atividades que favoreçam a uniformidade assistencial; ao paciente, quando este não está preparado emocionalmente para receber informações ou prefere recebê-las do médico; à política institucional, quando a instituição não favorece a participação do enfermeiro no processo, requisitando supervisão médica em detrimento da colaboração entre médicos e enfermeiros; aos profissionais, quando o enfermeiro não detém o conhecimento e o treinamento necessários ou apresenta pouca experiência profissional; à gestão institucional, quando a instituição não provê recursos adequados ou não apoia os enfermeiros para realizar a atividade ${ }^{(13)}$.

$\mathrm{O}$ segundo constructo analisado referiu-se à percepção do enfermeiro quanto ao respeito ao direito do paciente, no que concerne à identificação pessoal enquanto submetido aos cuidados de saúde e de Enfermagem. Os participantes desta pesquisa perceberam-se respeitando frequentemente os pressupostos de identificação pessoal, tendo esse constructo obtido média 4,63, com desvio-padrão próximo a zero $(0,03)$. 0 Código de Ética dos Profissionais de Enfermagem deixa claro que "o respeito aos direitos humanos é inerente ao exercício da profissão, o que inclui direitos (...) à dignidade e a ser tratada sem distinção (...)”(10). Esta referência no texto da Resolução ${ }^{(10)}$ fundamenta a razoabilidade para o profissional de enfermagem identificar-se ao paciente que se encontra sob cuidados e para chamá-lo por seu nome e sobrenome, uma vez que tais atitudes garantem um posicionamento respeitoso diante a dignidade da pessoa. Ao respeitar a dignidade da pessoa, reconhece-se sua unicidade, sua individualidade e sua biografia, conhecidas no seio da sociedade civil a partir da atribuição de um nome e de um sobrenome.

A Carta dos Direitos dos Usuários de Saúde ${ }^{(16)}$ assegura aos cidadãos o direito básico ao ingresso digno nos sistemas de saúde públicos e privados. Um de seus princípios assegura o atendimento acolhedor, livre de qualquer forma de discriminação. Para que se cumpra este princípio, a norma determina que se garanta ao paciente: “A 
identificação do nome e do sobrenome, devendo existir em todo documento de identificação do usuário um campo para registrar o nome pelo qual prefere ser chamado", e que os "profissionais que se responsabilizem por sua atenção estejam identificados por meio de crachás visíveis e legíveis"(16).

A atitude do enfermeiro de chamar o paciente pelo nome demonstra o reconhecimento de sua individualidade e singularidade. Um tratamento adequado, baseado no respeito é importante e imprescindível para criar um vínculo com a pessoa a ser cuidada ${ }^{(17)}$, é o primeiro passo para se construir uma relação entre o paciente e o enfermeiro fundamentado num diálogo respeitoso envolvendo compromisso e valorização com o ser humano. A atitude de chamar o paciente pelo nome está relacionado ao desenvolvimento de uma assistência humanizada. O diálogo, as informações oferecidas ao enfermo e a identificação do doente pelo nome são descritas como ações que levam à humanização do cuidado ${ }^{(18)}$.

Dando sequência à análise, o terceiro constructo do instrumento que compõe o respeito aos direitos dos pacientes denomina-se - respeito à autonomia do cliente. As questões relacionadas a este constructo fazem referência à aquisição do consentimento dos doentes pelos enfermeiros, para submeterem-se a ações terapêuticas e a procedimentos de enfermagem. A atitude dos enfermeiros de solicitar consentimento para realizar ações terapêuticas e procedimentos de enfermagem obteve média de 4,62 e 4,68, respectivamente. A ação de permitir acesso ao prontuário obteve média de 4,63.

O consentimento informado é a ferramenta básica que consolida a autonomia do paciente ${ }^{(19)}$. Ao solicitar ao paciente o consentimento para realizar atividades relacionadas ao cuidado, o enfermeiro reconhece neste a sua capacidade de tomar decisões autônomas relacionadas à saúde e bem-estar ${ }^{(20)}$. Para isso, os pacientes necessitam receber informações sobre sua condição clínica, a opções terapêuticas disponíveis e os riscos e benefícios das intervenções propostas ${ }^{(19)}$.

Para que o consentimento expresse o real exercício da autonomia, o paciente deve demonstrar capacidade de compreender as informações, de decidir sem que haja interferência de outros e de forma intencional conceder autorização ao profissional ${ }^{(8)}$. Por se caracterizar como um processo comunicativo, a aquisição de consentimento requer que o profissional tenha habilidades de comunicação que possam ajudar o paciente a compreender sua situação clínica mesmo diante do seu estado de fragilidade e vulnerabilidade enquanto pessoa doente ${ }^{(19)}$.

Os resultados deste estudo demonstraram que os enfermeiros percebem-se respeitando o direito do paciente de consentir ações terapêuticas e procedimentos de enfermagem. Ainda são poucos os estudos que apresentam dados sobre o engajamento do enfermeiro na aquisição do consentimento ${ }^{(20)}$. Estudos realizados com pacientes, apontam que estes nem sempre se sentem bem informados no momento de tomar decisões ${ }^{(21)}$ ou perceberam a atitude do profissional como mera formalidade ${ }^{(19)}$.

Considerando os pressupostos para aquisição do consentimento, é inegável a relação que existe entre os constructos "informação para tomada de decisão" e "respeito à autonomia do paciente" do instrumento de coleta de dados, uma vez que este último é precedido pelo primeiro. Nesse sentido, para verificar se existe diferença significativa de performance dos participantes entre esses dois constructos, foi aplicado o teste de Wilcoxon. Assim, constatou-se que o constructo "respeito à autonomia do cliente" é percebido como mais exercido em relação à "identificação pessoal” ( $p=0,001)$ e à "informação para tomada de decisão" $(p<0,001)$. 
Diante deste resultado, há que se questionar qual a compreensão dos participantes acerca do termo "consentir", uma vez que a prática percebida não harmoniza com os pré-requisitos exigidos pela teoria existente. Todavia, a literatura pode amparar a compreensão a respeito dessa situação. Existe, na área da saúde, um conceito bastante difundido: o consentimento implícito, o qual se sustenta na expectativa dos profissionais de que o paciente está a espera de que determinados procedimentos sejam realizados enquanto ele se encontra sob cuidados de saúde. Por essa razão, o ato de consentir limita-se a realizar uma pergunta específica ao paciente e esperar sua permissão, fornecendo uma explicação mínima acerca das ações que serão desenvolvidas ${ }^{(22)}$.

Um estudo(23) sobre o conceito e o uso do consentimento implícito na realização de procedimentos de enfermagem apontou que a maioria dos enfermeiros entrevistados o utilizava antes de realizar procedimentos de enfermagem, e consideravam a não objeção do paciente à continuidade dos cuidados como uma forma de consentir. Um dos motivos para utilizar o consentimento implícito é a tolerância do enfermo à falta de informação. A postura conformada do doente leva ao não questionamento sobre os procedimentos e, por não serem questionados, os enfermeiros limitam-se a fornecer algumas informações que julgam adequadas. 0 consentimento implícito tem sido usado para realizar cuidados de enfermagem de rotina, sendo visto como um consenso geral na prática de enfermagem ${ }^{(22)}$, o que se torna preocupante, uma vez que tal ação pode não beneficiar a autonomia do paciente e o seu direito de consentir ou de recusar procedimentos de enfermagem.

Os enfermeiros são os profissionais em quem os pacientes mais confiam para responder questionamentos e sanar dúvidas ${ }^{(19)}$, logo, o enfermeiro destaca-se como elemento fundamental na realização do processo de consentimento e, por conseguinte, no incentivo à autodeterminação do paciente. O contato continuado e prolongado com o doente, pode ser utilizado como oportunidade para reforçar com este a existência de seus direitos e sua prerrogativa pessoal de autodeterminação.

O detalhamento da percepção do enfermeiro neste estudo, viabilizou não somente a verificação de quais fundamentos do respeito aos direitos dos pacientes são percebidos como mais respeitados, mas também fez despontar o questionamento acerca do conhecimento teórico que os enfermeiros possuem sobre os conceitos e a operacionalização destes fundamentos. Ou seja, os resultados encontrados podem ter sofrido interferência, em alguma medida, da ausência de clareza sobre os conceitos que embasam os fundamentos do respeito aos direitos do paciente, ou até mesmo do próprio dessaber dos enfermeiros. A literatura utilizada evidenciou diversas situações em que tais conceitos foram mal compreendidos ou estavam sendo operacionalizados de maneira incoerente. Esse fato deve ser considerado como ponto limitante deste estudo.

\section{CONCLUSÃO}

Verificou-se neste estudo que os enfermeiros se percebem atuando ante ao respeito dos direitos dos pacientes em todos os fundamentos avaliados. Este resultado auxilia a ratificar o valor moral da profissão de enfermagem e reforça quais atitudes e comportamentos éticos são salutares na assistência à saúde e quais necessitam ser implementados, a fim de que os pacientes tenham seus direitos respeitados.

Outro fato importante é a necessidade de aprofundar o conhecimento acerca do valor do cuidado enquanto instrumento ético. Espera-se que este estudo conduza a uma reflexão a respeito de atitudes adotadas na prática 
clínica pelos enfermeiros, bem como ao interesse da comunidade científica em aprofundar investigações na área de Ética em Enfermagem e Bioética, no sentido de ampliar o referencial teórico nestas temáticas.

A limitação identificada pode ser superada com a realização de estudos complementares que utilizem desenhos metodológicos qualitativos capazes de evidenciar o conhecimento dos enfermeiros acerca dos fundamentos dos direitos dos pacientes e a aplicabilidade prática na prestação de cuidados, ajudando a elucidar questionamentos e fornecendo mais conteúdo a este debate.

\section{REFERÊNCIAS}

1. Saraiva AMP. Acesso à tecnologia biomédica: perspectiva bioética dos enfermeiros portugueses. Rev. Bioét. [Internet]. 2014 [acesso em: 01 nov. 2018];22(1):161-170. Disponível em: http://dx.doi.org/10.1590/S1983-80422014000100018

2. Nunes MI, Anjos MF. Diretivas antecipadas de vontade: benefícios, obstáculos e limites. Rev. Bioét. [Internet]. 2014 [acesso em: 01 nov. 2018];22(2):241-251. Disponível em: http://dx.doi.org/10.1590/1983-80422014222005

3. Sanchez-Gonzales M, Herreros B. La bioética em la prática clínca. Rev Med Inst Mex Seguro Soc [Internet]. 2015 [acesso em: 01 nov. 2018];53(1):66-73. Disponible em: http://www.medigraphic.com/pdfs/imss/im-2015/im151n.pdf

4. Bayer GLA. Enfermería y el respeto a la autonomia en el ambito hospitalario: entre la sobrepoteccion y el descuido. Av.enferm [Internet]. 2013 [acesso em: 27 nov. 2016];21(1):126-131. Disponible en: http://www.scielo.org.co/pdf/aven/v31n1/v31n1a12.pdf 5. Sorokin P, Actis AM, Outomuro D. Comités de ética asistencial: de los grandes dilemas a los nuevos desafíos. Rev. Bioét. [Internet]. 2016 [acesso em: 02 nov. 2018];24(1):91-97. Disponible em: http://dx.doi.org/10.1590/1983-80422016241110 6. Nogueira FM. Aspectos éticos da inovação em saúde em Portugal. Rev. Bioét. [Internet]. 2016 [acesso em: 02 nov. 2018];24(1):83-90. Disponível em: http://dx.doi.org/10.1590/1983-80422016241109

7. Gatsman C. Dignity-enhacing nursing care: a foundational ethical framework. Nurs Ethics [Internet]. 2013 [acesso em: 28 jul. 2016];20(2):142-149. Available from: http://dx.doi.org/10.1177/0969733012473772

8. Beauchamp TL, Childress JF. Principles of biomedical ethics. 7th ed. New York: Oxford University Press; 2013.

9. Ferri P, Muzzalupo J, Di Lorenzo R. Patients' perception of dignity in an Italian general hospital: a cross-sectional analysis. BMC Health Serv Res [Internet]. 2015 [acesso em: 03 nov. 2018];15(41):2-8. Available from: https://dx.doi.org/10.1186\%2Fs12913-0150704-8

10. Resolução Conselho Federal de Enfermagem no 564, de 06 de dezembro de 2017 (BR) [Internet]. Código de ética dos profissionais de Enfermagem. [acesso em 25 out. 2018]. Disponível em: http://www.cofen.gov.br/resolucao-cofen-no$\underline{5642017 \text { 59145.html }}$

11. Alves PC, Lunardi VL, Lunardi GL, Lunardi Filho WD. A percepção das enfermeiras acerca da sua atuação ante os direitos dos clientes. Rev. esc. enferm. USP [Internet]. 2008 [acesso em: 26 jan. 2016];42(2):242-248. Disponível em:

http://dx.doi.org/10.1590/S0080-62342008000200005

12. Tariman D, Szubski KL. The evolving role of the Nurse during the cancer treatment decision-making process: a literature review. CJON [Internet]. 2015 [acesso em: 15 mar. 2017];19(5):548-556. Available from: http://dx.doi.org/10.1188/15.CJON.548-556 13. McCarter SP, Tariman JD, Spawn N, Mehmet[i E, Bishop-Royse J, Garcia I, et al. Barriers and promoters to participation in the era of shared treatment decision-making. WJNR [Internet]. 2016 [acesso em: 18 mar. 2017];38(10):1282-1297. Available from: http://dx.doi.org/10.1177/0193945916650648

14. Tariman JD, Mehmeti E, Spawn N, McCarter SP, Bishop-Royse J, Garcia I, et al. Oncology nursing and shared decision making for cancer treatment. CJON [Internet]. 2016 [acesso em: 18 mar. 2017];20(5):560-563. Available from:

http://dx.doi.org/10.1188/16.CJON.560-563

15. Baliza MF, Bousso RS, Poles K, Santos MR, Silva L, Paganini MC. Fatores que influenciam os enfermeiros de Unidades de Terapia Intensiva nas decisões de final de vida. Rev. esc. enferm. USP [Internet]. 2015 [acesso em: 28 mar. 2017];49(4):572-579. Disponível em: http://dx.doi.org/10.1590/S0080-623420150000400006

16. Ministério da Saúde. Conselho Nacional de Saúde. Carta dos direitos dos usuários da saúde [Internet]. Brasília: Ministério da Saúde, 2011 [acesso em: 28 mar. 2017]. Disponível em:

http://bvsms.saude.gov.br/bvs/publicacoes/cartas direitos usuarios saude 3ed.pdf

17. Branco LASC, Maia NMFS, Lima LAA. A construção do vínculo enfermeiro-cliente pelo diálogo no ambiente hospitalar. Rev Enferm UFPI [Internet]. 2016 [acesso em: 04 nov. 2018];5(3):30-35. Disponível em: https://doi.org/10.26694/reufpi.v5i3.5436 18. Vila VS, Rossi LA. The cultural meaning of humanized care in intensive care units: a lot is said about it, but little is experienced. Rev. Latino-am. Enfermagem [Internet]. 2002 [acesso em: 10 abr. 2017];10(2):137-144. Avaliable from:

http://dx.doi.org/10.1590/S0104-11692002000200003 
19. Busquets M, Cais J. Informed consent: A study of patients with life-threatening illnesses. Nurs Ethics [Internet]. 2015 [acesso em: 04 nov. 2018];24(4):430-440. Avaliable from: http://dx.doi.org/10.1177/0969733015614880

20. Giordano NA, Axson SA, Hermann RM, Ulrich CM. Evaluating nurse understanding and participation in the informed consent process. Nurs Ethics [Internet]. 2017 [acesso em: 04 nov. 2018]:1-12. Avaliable from:

http://dx.doi.org/10.1177/0969733017740175

21. Susilo AP, Van Dalen J, Chenault N, Scherpier A. Informed consent and nurses' roles: A survey of Indonesian practitioners. Nurs Ethics [Internet]. 2014 [acesso em: 03 nov.2018];21(6):684-694. Avaliable from: http://dx.doi.org/10.1177/0969733014531524 22. Cole CA. Implied consent and nursing practice: ethical or convenient? Nurs Ethics [Internet]. 2012 [acesso em: 14 abr.

2017];19(4):550-557. Avaliable from: http://dx.doi.org/10.1177/0969733011436028

23. Aveyard H. Implied consent prior to nursing care procedures. JAN [Internet]. 2002 [acesso em 25 mar. 2017];39(2):201-207. Avaliable from: http://dx.doi.org/10.1046/j.1365-2648.2002.02260.x. 